\title{
Intelligent Modeling of Permeate Flux during Membrane Clarification of Pomegranate Juice
}

\author{
Elham Moradi Avarzaman ${ }^{1}$, Payam Zarafshan ${ }^{2 *}$, Hossein Mirsaeedghazi $^{3}$, Behzad Alaeddini $^{1}$ \\ 1- Department of Food Science, Nour Branch, Islamic Azad University, Nour, Iran. \\ 2- Department of Agrotechnology, College of Abouraihan, University of Tehran, Pakdasht, Tehran, Iran. \\ 3- Department of Food Technology, College of Abouraihan, University of Tehran, Pakdasht, Tehran, Iran.
}

\section{A B S T R A C T}

Background and Objectives: One of the problems in juice membrane clarification is the accumulation and deposition of rejected compounds on membrane surfaces or inside its pores which results in a membrane fouling.

Materials and Methods: Several parameters can have influence on fouling in one hand and prediction of juice permeates flux during the membrane processing is of importance in industrial applications on the other hand. Therefore, providing a model able to predict the permeate flux having the value of effective input parameters seems to be useful. In this regard, several artificial methods can be used. In contrast, the Fuzzy Inference System (FIS) has been proposed as a reliable and appropriate machine learning method to predict the output parameter with acceptable performance. In this study, a FIS will be used to model the permeate flux based on five input variables: the transmitted membrane pressure, feed flow rate, processing time, membrane pore size, and membrane type. For this purpose, a fuzzy system is trained using the laboratory data and then, appropriate membership functions for the input and output variables and fuzzy rules are extracted for the proper prediction of permeate flux.

Results: Results were shown that the normalized mean squared errors for the prediction of permeate flux in the membrane processing are 0.0055 and 0.0081 for the Mixed Cellulose Ester (MCE) and the Polyvinylidene Flouride (PVDF) membranes, respectively.

Conclusions: In total, the fuzzy inference model which is presented in this study has been able to predict the pomegranate juice permeate flux with an acceptable error compared with the laboratory data.

Keywords: Pomegranate Juice, Clarification, Membranes Process, Fuzzy Inference System

\section{Introduction}

Pomegranate (Punica Granatum L.) is a popular fruit which is widely cultivated throughout the Middle East, Africa, the Indian subcontinent, Central Asia, and United states, (1). Its popularity is mainly because of its phenolic compounds such as flavonoids and phenolic acid, which can counteract different types of cancer in the human, (2). Despite nutritional properties, pomegranate juice has a turbid appearance which makes its preservation and concentration difficult in processing steps. Today, the use of membrane systems has become a common technique in the clarification of juices because of their advantages in low energy consumption due to not having phase changes, variation in membrane shape, its size, high separation efficiency for diluted solutions, little demand to additives, and the ease of use compared to the traditional methods, (3). Microfiltration is one of the important membrane processes based on the physical separation in which ingredients are separated according to their sizes. One 
of the problems in juice microfiltration is the accumulation and deposition of rejected compounds on membrane surfaces or inside its pores which results in the membrane fouling. This phenomenon is the most important reason why the membrane clarification has not been yet considered as an appropriate industrialized juice clarification method. Various parameters can affect this phenomenon including the feed temperature, pressure of feed transmission through membrane, flow speed, membrane material, and size of the membrane pores, (4).

Since an understanding about relationships between these parameters and the permeate flux is valuable in the membrane technology, providing a model able to predict the permeate flux by having the input parameters seems to be useful. These models can play an important role in the simulation and optimization of membrane systems providing efficient and economical designs in juice clarification processes, (5). Several methods have been developed to model the food engineering processes including logistic regressions and machine learning methods. Regression models are suitable where the output parameter is a function of at most two variables. Regression modelling of problems with more than three input parameters usually results in complex nonlinear equations with extra coefficients. Machine learning methods are based on the computer pattern recognition and usually provide a black-box which user cannot use it without a computer. In recent years, hundreds of researches have been published about the application of machine learning methods in the agricultural engineering, (6). Artificial neural network is one of the most applicable techniques in the machine learning in which the neurons are responsible to learn the nonlinear relationship between input and output parameters, (7). Furthermore, several researchers have utilized artificial neural networks for the prediction of permeate flux in the membrane clarification of mosambi juice, (8), milk (9-10), and red plum juice, (11). Among artificial intelligence methods, Fuzzy Inference Systems (FISs) have become a popular method to design robust prediction models because of their ability in transferring the human skills into linguistic rules, (12). Instead of being black-box, these models provide membership functions for input and output parameters and fuzzy rules to properly relate input parameters to outputs, (13). Mehraban
Sangtarash et al., (14), assessed the quality of raw milk using the fuzzy logic. In their study, it has been tried to use expert opinions and triangular membership functions to model the experimental data of raw milk quality. They showed that the FIS compared to the direct determination of the quality of raw milk can result in the acceptable performance. In a research carried out by Ebadati and Vahaji, (15), several applications of fuzzy logic are studied including the quality assessment of raw milk and grading of apple fruits regarding to its shape and size. They showed that fuzzy sets are designed especially for problems with uncertainties which linguistic parameters can analyze quantitative problems. Harris, (16), used the fuzzy logic for grading raw milk in terms of quality and health characteristics. In his paper, the hygienic quality and the compositional quality parts of the standard have been recast by fuzzy logic inference system to form a structured judgment system for grading both the hygienic and compositional quality of milk. Mazloumzadeh et al., (17), used Mamdani FIS to classify the productive trees based on yield, fruit length and visual appearance, and to produce a tree total quality map for each grove. Da Silva et al., (18), reported that conventional mathematical models used to represent the crossflow filtration process have some limitations in relation to the identification and generalization of the system behavior. They developed a fuzzy logic system to overcome the problems usually found in the conventional mathematical models. They figured out imprecisions and uncertainties associated with the experimental measurements made on the system are automatically incorporated in the fuzzy approach. Cavero et al., (19), developed a fuzzy logic model for classification and control of mastitis for cows milked in an automatic milking system. They showed that fuzzy logic is a useful tool to develop a detection model for mastitis. They also reported that in this specific case, a noticeable decrease in the error rate can be made possible by means of more informative parameters. Prasad and Nath, (20), applied response surface methodology and fuzzy techniques for the development of sugarcane juice based on the lime beverage. Both the techniques were compared for the adequacy of optimization and applicability in the beverage industry. The results of fuzzy technique in agreement with the response surface methodology based experimentation results. Therefore, they concluded that fuzzy technique could be used for the 
optimization and development of beverage formulation. Jaya and Das, (21), used the fuzzy logic analysis to find out the best of three market mango drinks. BrimA index (a criterion for acceptance of fruit juice) and the total solid ( $\mathrm{kg}$ per $\mathrm{kg}$ drink) of reconstituted mango drink were considered as the input parameters of the fuzzy model where the subjective quality of the drinks was the model output. Sun and Qi, (22), adopted a fuzzy mathematical method in the sensory quality evaluation of ginger wine which is prepared by the superfine grinding, enzymatic hydrolysis, ultrafiltration, and chitosan treatment. Results showed that using the fuzzy model can properly predict the quality of ginger wine. Yang, (23), proposed a fuzzy comprehensive evaluation model for the mulberry wine quality. The influencing factors of mulberry wine quality including color, flavor, taste, style and so on were investigated comprehensively by the use of a weight distribution method and a multiply operator method. His model was able to improve the traditional sensory evaluation and diminished manmade subjective effects on the evaluation.

So, the objective of this study is to provide a fuzzy inference system to model the pomegranate juice permeate flux based on four input variables: pressure, flow rate, pore size and membrane type. To this end, laboratory experiments are carried out to investigate the effects of variation in input parameters' values on permeate flux. Initial membership functions of problem variables and fuzzy rules are selected based on past researches and then, a modification algorithm is used to revise the membership functions based on training the model using the laboratory data to obtain a robust and applicable fuzzy model.

\section{Materials and Methods}

Pomegranate Juice: Sweet-sow pomegranate was provided from a research pomegranate garden (Saveh, Iran). Juice extraction was prepared from fresh pomegranate seeds. The juice was manually extracted, and large particles such as peel were removed using a mesh filter (No. 9). Obtained juice was stored at -25 ${ }^{\circ} \mathrm{C}$ until the experiments in polyethylene terephthalate packages.

Membrane Processing Method: A cross-flow membrane unit with a flat sheet module in the batch mode was used at the laboratory scale according to Nourbakhsh et al., (11). Mixed cellulose ester (MCE) membranes with pore sizes of $0.22,0.1$ and $0.025 \mu \mathrm{m}$ (Millipore, United States) and Polyvinylidene
Flouride (PVDF) membrane with pore size of 0.22 $\mu \mathrm{m}$ (Millipore, United States) were used in this study. The permeate flux of clarified juice was determined as, (11):

$\mathrm{J}_{\mathrm{p}}=\frac{\Delta \mathrm{m}}{\mathrm{A} \times \mathrm{t}}$

where $\Delta m$ is the permeate weight $(\mathrm{kg}), A$ is the effective membrane area $\left(\mathrm{m}^{2}\right)$, and $t$ is the time (h). To investigate the effects of input parameters including the transmitted membrane pressure, feed flow rate, membrane pore size and membrane type on the pomegranate juice permeate flux, several experiments are carried out in the laboratory to provide training samples for the fuzzy model. To this end, a dataset of samples (patterns) is obtained from the membrane clarification treatments of pomegranate juice. The input variables of the model are transmitted the membrane pressure $(0.5,2$, and 5 bars), feed flow rate $(0.095$ and $0.533 \mathrm{~m} / \mathrm{s})$, membrane pore size $(0.025,0.1$ and $0.22 \mu \mathrm{m})$, membrane type (MCE and PVDF) and time (during $120 \mathrm{~min}$ of processing with certain intervals). The model output is the permeate flux of pomegranate juice. All experiments are run with three replications and mean values are recorded.

Fuzzy Inference Modelling: Fuzzy Inference System is one of the machine learning methods which its acceptable performance in the prediction of output parameters is reported by hundreds of researches in food engineering problems. The flowchart of machine learning methods in the prediction of juice permeate flux is shown in Figure 1. The first step in the fuzzy modeling is the feature normalization of the samples. The features of all samples are normalized using the Eq. (2) to make it possible to compare the error parameters of this model with other models introduced in last researches. Eq. (2) maps all values for each feature between 0 and 1 as:

$x_{n}=\frac{x_{o}-x_{\min }}{x_{\max }-x_{\min }}$

where $x_{n}$ is the sample's feature value after normalization, $x_{o}$ is the raw feature value, and $x_{\min }$ and $x_{\max }$ are the lowest and highest values of each features, respectively. The first step in designing a fuzzy logic model is to determine membership functions of the input and output parameters. Since it is not possible to assign membership functions based on linguistic terms to the membrane type, a fuzzy model is designed for the MCE membrane and another one for PVDF separately. 


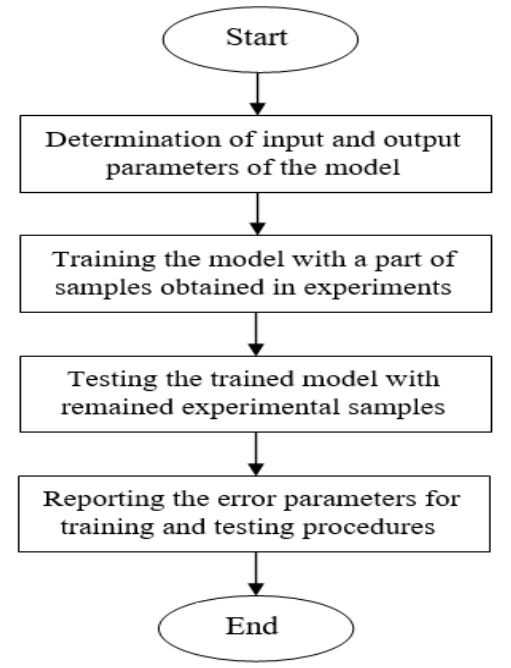

Figure 1. The flowchart of machine learning methods in parameter prediction
In contrast with black-box methods, it is mandatory that expert scientists provide membership functions and fuzzy rules based on the nature of the problem, the variation range of the features (its variance and center), and the relations between input and output parameters. To this end, Table 1 shows the proposed membership functions for each fuzzy model. As seen from this table, each variable possessed three membership functions with appropriate linguistic names including "small, medium, and large" or "low, medium, and high". From this table, all membership functions are considered Gaussian functions defined with center and variance values.

Table 1. Proposed membership functions for input and output parameters of the model

\begin{tabular}{|c|c|c|c|c|}
\hline \multirow{2}{*}{ Membrane type } & \multirow{2}{*}{ Parameter } & \multirow{2}{*}{ Parameter level } & \multicolumn{2}{|c|}{ Membership function characteristics } \\
\hline & & & Center & Variance \\
\hline \multirow{15}{*}{ MCE } & \multirow{3}{*}{ Pore size } & Small & 0.10 & 0.30 \\
\hline & & Medium & 0.50 & 0.30 \\
\hline & & Large & 0.90 & 0.30 \\
\hline & \multirow{3}{*}{ Flow rate } & Low & 0.01 & 0.10 \\
\hline & & Medium & 0.50 & 0.10 \\
\hline & & High & 0.90 & 0.10 \\
\hline & \multirow{3}{*}{ Pressure } & Low & 0.10 & 0.20 \\
\hline & & Medium & 0.50 & 0.20 \\
\hline & & High & 0.90 & 0.20 \\
\hline & \multirow{3}{*}{ Time } & Low & 0.20 & 0.30 \\
\hline & & Medium & 0.50 & 0.30 \\
\hline & & High & 0.80 & 0.30 \\
\hline & \multirow{3}{*}{ Permeate flux } & Low & 0.10 & 0.20 \\
\hline & & Medium & 0.20 & 0.30 \\
\hline & & High & 0.50 & 0.40 \\
\hline \multirow{12}{*}{ PVDF } & \multirow{3}{*}{ Flow rate } & Low & 0.20 & 0.10 \\
\hline & & Medium & 0.50 & 0.10 \\
\hline & & High & 0.80 & 0.10 \\
\hline & \multirow{3}{*}{ Pressure } & Low & 0.20 & 0.15 \\
\hline & & Medium & 0.40 & 0.15 \\
\hline & & High & 0.80 & 0.15 \\
\hline & \multirow{3}{*}{ Time } & Low & 0.20 & 0.25 \\
\hline & & Medium & 0.50 & 0.25 \\
\hline & & High & 0.80 & 0.25 \\
\hline & \multirow{3}{*}{ Permeate flux } & Low & 0.20 & 0.10 \\
\hline & & Medium & 0.30 & 0.20 \\
\hline & & High & 0.60 & 0.30 \\
\hline
\end{tabular}


The reason here is the smooth variation of output variable due to the changes of input variables in one hand and requiring to only two parameters for the fully definition. The values of Gaussian parameters presented in Table 1 are initial values and need to be modified for the better prediction of permeate flux. Later in this section, the proposed algorithm for modifying the values of these parameters is studied. The second step in fuzzy modeling is the determination of fuzzy rules between input and output variable. Analyzing the relationships between input and output variables of fuzzy models is necessary for the one who wants to determine fuzzy rules. Transmitted membrane pressure is the driving force for permeation, but the flux increases with pressure up to a certain limiting value. Furthermore, increasing of cross-flow rate would enhance wall shear stress on the membrane surface. Higher wall shear force is helpful to reduce the membrane pore blockage and fouling, (3). Despite the several benefits of membrane clarification, the performance of this operation is affected by the declining permeate flux with time, (24). In addition, greater pore size can result in the greater permeate flow through the membrane. Several researches have studied the effects of various parameters on the permeate flux in the membrane clarification, (25-29). According to the literature, six fuzzy rules can be determined in the fuzzy modeling according to Table 2.

An algorithm is required to modify the parameters of membership functions presented in Table 1 to obtain the proper performance in the prediction of permeate flux. To this end, an iterative algorithm is proposed to modify the parameters of membership functions. From the recorded experimental data in the laboratory, $80 \%$ of samples are randomly selected to train the fuzzy model using the algorithm of Figure 2. Centroid method is used for the defuzzification in this algorithm. The division of samples for training and testing the fuzzy model is shown in Table 3.

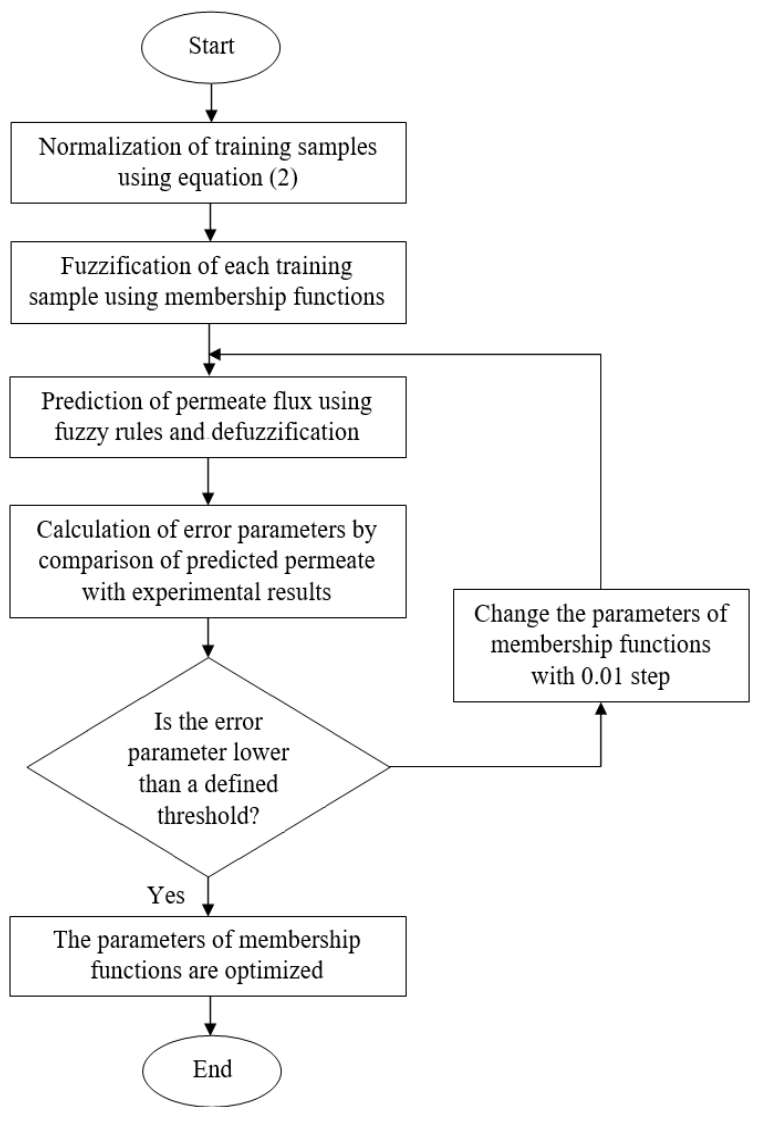

Figure 2. Proposed algorithm for parameters modification of membership functions

Table 2. The proposed fuzzy rules for prediction of the output variable

\begin{tabular}{ccccccc}
\hline Membrane type & Rule No. & Pore size & Flow rate & Pressure & Time & Permeate flux \\
\hline \multirow{3}{*}{ PVDF } & 1 & - & low & low & high & low \\
& 2 & - & medium & medium & medium & medium \\
& 3 & - & high & high & low & high \\
MCE & 4 & small & low & low & high & low \\
& 5 & medium & medium & medium & medium & medium \\
& 6 & large & high & high & low & high \\
\hline
\end{tabular}

Table 3. Number of used data in training and testing the fuzzy model

\begin{tabular}{lcc}
\hline \multirow{2}{*}{ Sample division } & \multicolumn{2}{c}{ Membrane type } \\
\cline { 2 - 3 } & PVDF & MCE \\
\hline Training 80\% & 96 & 160 \\
Test 20\% & 24 & 40 \\
\hline
\end{tabular}


Performance Evaluation Criteria: The performance of the designed fuzzy model is evaluated based on error parameters including the Mean Square Error (MSE), Root Mean Square Error (RMSE), and Mean Absolute Error (MAE) for train and test samples, separately. These statistical parameters can be calculated using, (30):

$$
\begin{aligned}
& M S E=\frac{1}{N} \sum_{i=1}^{n}\left(x_{\text {predicted }}-x_{\text {measured }}\right)^{2} \\
& R M S E=\sqrt{\frac{1}{N} \sum_{i=1}^{n}\left(x_{\text {predicted }}-x_{\text {measured }}\right)^{2}} \\
& M A E=\frac{1}{N} \sum_{i=1}^{n}\left|\left(x_{\text {predicted }}-x_{\text {measured }}\right)\right|
\end{aligned}
$$

where $N$ is the number of samples, $x_{\text {predicted }}$ is the fuzzy model output for the permeate flux and $x_{\text {measured }}$ is the measured value of permeate flux in the laboratory. The better performance of the proposed fuzzy model is attained when these error parameters are low.

\section{Results and Discussion}

Modified Membership Functions: Table 4 shows the modified membership functions for input and output parameters of the proposed fuzzy model. In contrast with black-box methods such as artificial neural networks and support vector machines, one can predict the permeate flux by having the membership functions and fuzzy rules. Therefore, there will be no need to solve complex mathematical equations or having computers to predict the output parameter. As can be seen in this table, all parameters of Gaussian functions are modified to improve the performance of

\begin{tabular}{|c|c|c|c|c|}
\hline \multirow{2}{*}{ Membrane type } & \multirow{2}{*}{ Parameter } & \multirow{2}{*}{ Parameter level } & \multicolumn{2}{|c|}{ Membership function characteristics } \\
\hline & & & Center & Variance \\
\hline \multirow{15}{*}{ MCE } & \multirow{3}{*}{ Pore size } & Small & 0.06 & 0.38 \\
\hline & & Medium & 0.65 & 0.09 \\
\hline & & Large & 0.95 & 0.17 \\
\hline & \multirow{3}{*}{ Flow rate } & Low & 0.00 & 0.07 \\
\hline & & Medium & 0.21 & 0.08 \\
\hline & & High & 0.40 & 0.07 \\
\hline & \multirow{3}{*}{ Pressure } & Low & 0.11 & 0.22 \\
\hline & & Medium & 0.32 & 0.30 \\
\hline & & High & 0.98 & 0.21 \\
\hline & \multirow{3}{*}{ Time } & Low & 0.22 & 0.21 \\
\hline & & Medium & 0.32 & 0.26 \\
\hline & & High & 0.41 & 0.21 \\
\hline & \multirow{3}{*}{ Permeate flux } & Low & 0.00 & 0.03 \\
\hline & & Medium & 0.12 & 0.05 \\
\hline & & High & 0.14 & 0.07 \\
\hline \multirow{12}{*}{ PVDF } & \multirow{3}{*}{ Flow rate } & Low & 0.00 & 0.13 \\
\hline & & Medium & 0.12 & 0.15 \\
\hline & & High & 0.98 & 0.27 \\
\hline & \multirow{3}{*}{ Pressure } & Low & 0.01 & 0.28 \\
\hline & & Medium & 0.49 & 0.15 \\
\hline & & High & 0.99 & 0.13 \\
\hline & \multirow{3}{*}{ Time } & Low & 0.21 & 0.17 \\
\hline & & Medium & 0.29 & 0.20 \\
\hline & & High & 0.42 & 0.33 \\
\hline & \multirow{3}{*}{ Permeate flux } & Low & 0.00 & 0.13 \\
\hline & & Medium & 0.02 & 0.15 \\
\hline & & High & 0.49 & 0.27 \\
\hline
\end{tabular}
the fuzzy model.

Table 4. Modified membership functions for input and output parameters of the model 
The relationship between model input and output parameters considering the modified membership functions and fuzzy rules is shown in Figure 3. Since the model have four input parameters, each figure represents the permeate flux as a function of two input parameters to simplify the illustration in a 3D Cartesian coordinates. The diagrams shown in Figure 3 are consistent with other researches on the effects of various parameters including transmitted membrane pressure, feed flow rate, processing time, membrane pore size and membrane type on permeate flux. All the input and output parameters vary in the determined range of [0-1]. This makes it possible to generalize the proposed fuzzy model for other laboratory conditions.
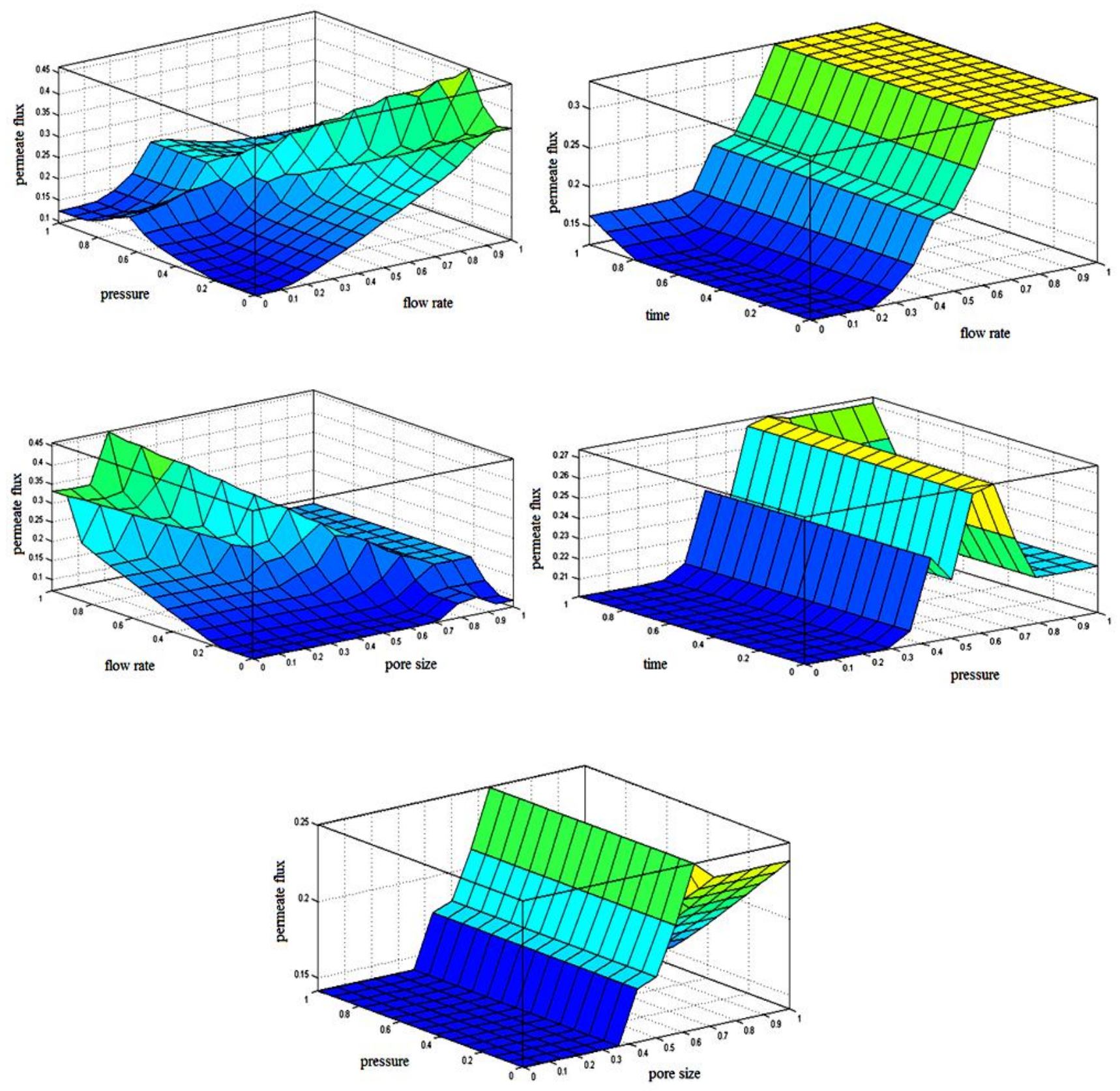

Figure 3. The relationship between model input and output parameters considering the modified membership functions and fuzzy rules 
Performance Evaluation of Fuzzy Model: Performance evaluation of fuzzy model for the MCE and PVDF membranes is shown in Table 5 based on error parameters. Since the values reported in this table are obtained after the normalization of output parameter, it is possible to compare these results with other researches which have studied different statistical and machine learning models in the prediction of juice permeate flux. The average training and testing MSE for two types of membrane are obtained as 0.0075 and 0.0085 , respectively. Nourbakhsh et al., (11), reported that these values are 0.0016 and 0.0021 , respectively using artificial neural networks for the red plum juice clarification. Although the artificial neural network usually results in lower error parameters, it cannot properly show the relationship between the input and output parameters since it is a block-box method. However, results shown in Table 5 state that the predictive accuracy of the fuzzy model is high and the model has been well trained. Therefore, the modified fuzzy inference system is a powerful tool to predict future values of permeation flux at various operating conditions through time which is essential in the designing membrane based on separation processes with the great saving in time and cost due to there is no need to carry out the large number of experiments to collect the filtration data.

Table 5. Performance evaluation of fuzzy model for MCE and PVDF membranes

\begin{tabular}{llllllll}
\hline \multirow{2}{*}{ Stage } & \multicolumn{3}{c}{ MCE } & & \multicolumn{3}{c}{ PVDF } \\
\cline { 2 - 4 } \cline { 6 - 8 } & MSE & RMSE & MAE & & MSE & RMSE & MAE \\
\hline Training & 0.005 & 0.069 & 0.100 & & 0.010 & 0.100 & 0.159 \\
Test & 0.005 & 0.071 & 0.123 & & 0.012 & 0.110 & 0.165 \\
\hline
\end{tabular}

Comparison of Experimental Data and Predicted

Data using the Fuzzy Model: A comparison of experimental data and predicted data using the fuzzy model is shown in Figures 4 and 5 for MCE and PVDF membranes, respectively. It can be found that there is a slight error between the experimental data (solid lines) and predicted curves (dots) at all experimental conditions. Furthermore, the diagrams in these figures confirm that the modified fuzzy model is able to simulate the non-linearity behavior of pomegranate juice permeate flux under experimental conditions properly. The trends of permeate flux in various conditions shows that the accumulation and fouling phenomena occurred at the initial stage of clarification and consequently, the flux decline rapidly. This is consistent with other researches results, $(11,25-28)$.

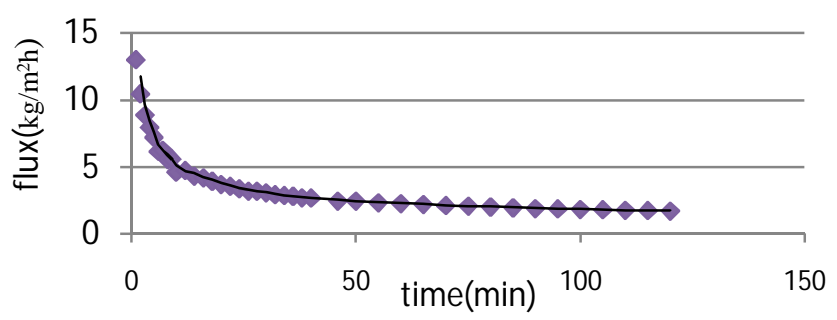

(a)

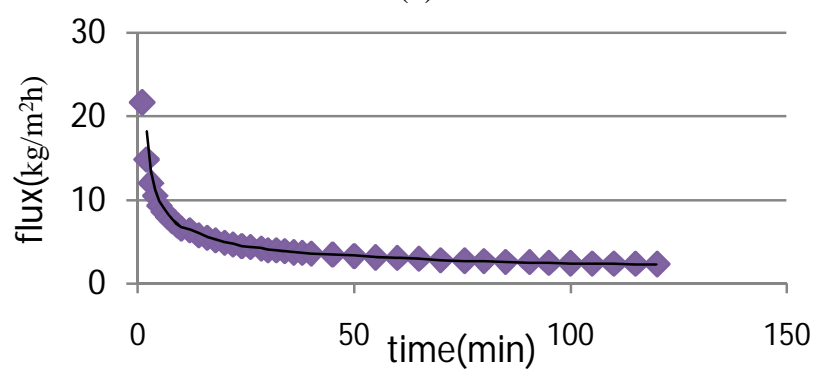

(b)

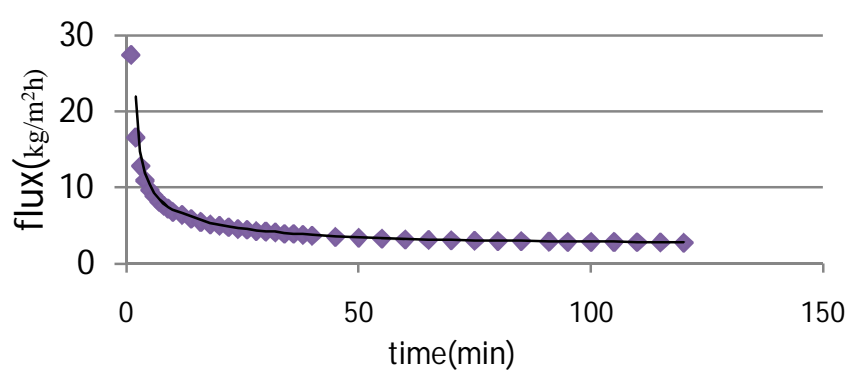

(c)

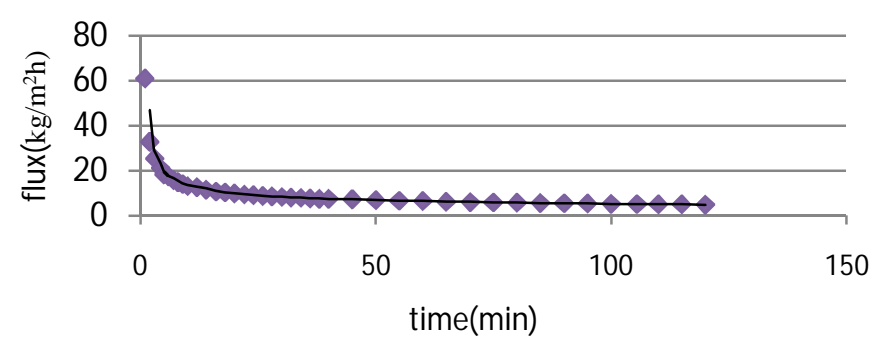

(d)

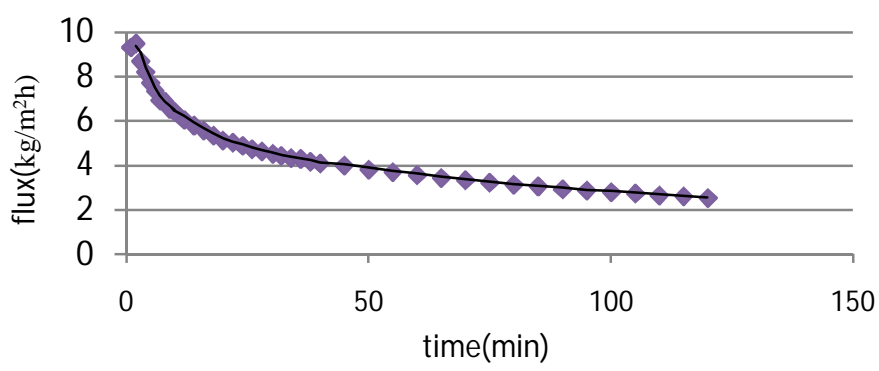

(e)

Figure 4. Measurement of permeate flux for different conditions using MCE membranes

a) flow rate of $0.095 \mathrm{~m} / \mathrm{s}$, pressure of $0.5 \mathrm{bar}$, and pore size of $0.1 \mu \mathrm{m}, \mathrm{b}$ ) flow rate of $0.095 \mathrm{~m} / \mathrm{s}$, pressure of $2 \mathrm{bar}$, and pore size of $0.1 \mu \mathrm{m}$, c) flow rate of $0.095 \mathrm{~m} / \mathrm{s}$, pressure of $2 \mathrm{bar}$, and pore size of $0.1 \mu \mathrm{m}, \mathrm{d}$ ) flow rate of $0.095 \mathrm{~m} / \mathrm{s}$, pressure of $5 \mathrm{bar}$, and pore size of $0.1 \mu \mathrm{m}, \mathrm{e}$ ) flow rate of $0.095 \mathrm{~m} / \mathrm{s}$, pressure of $0.5 \mathrm{bar}$, and pore size of $0.025 \mu \mathrm{m}$. 


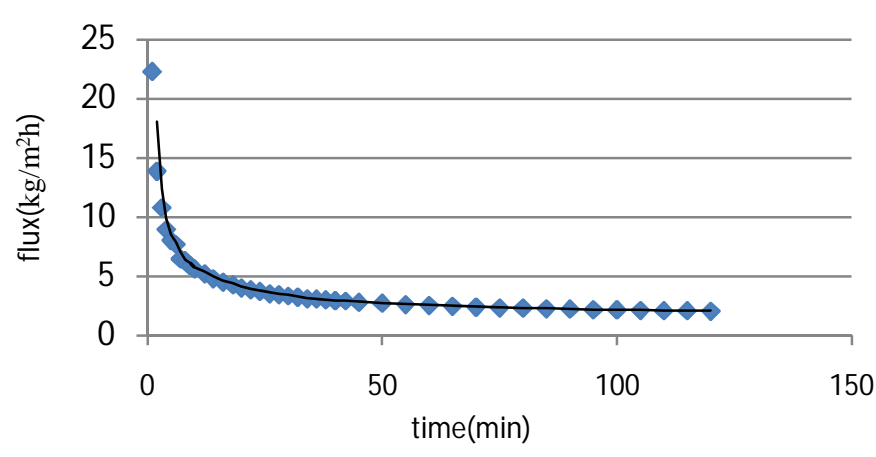

(a)

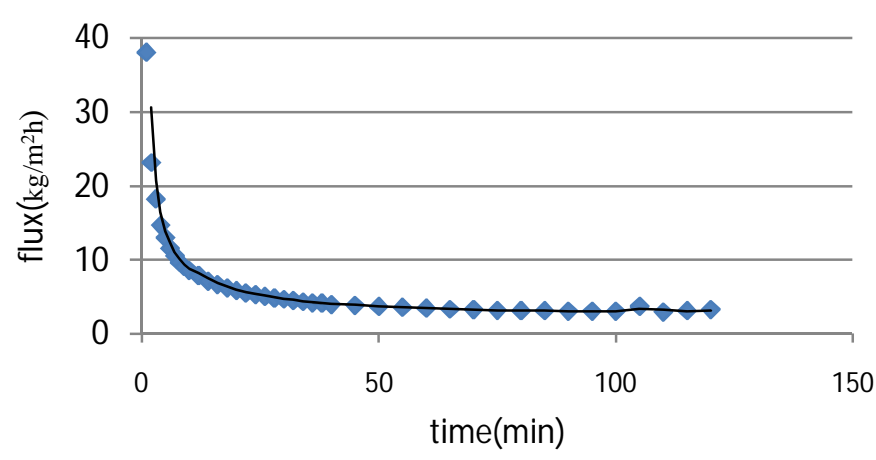

(b)

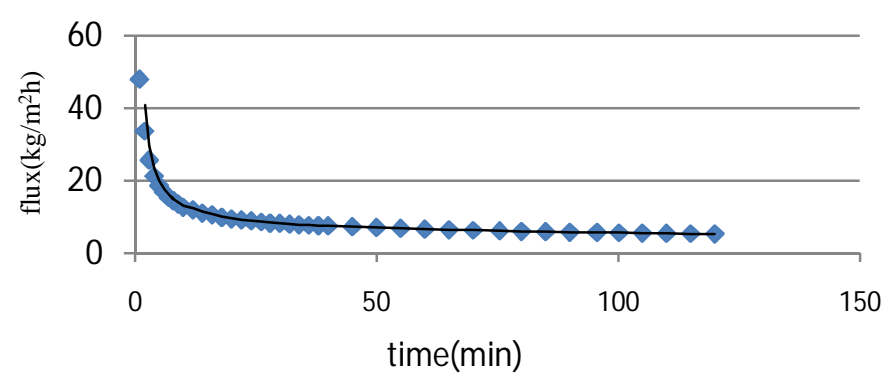

(c)

Figure 5. Measurement of permeate flux for different conditions using PVDF membranes,

a) flow rate of $0.095 \mathrm{~m} / \mathrm{s}$, pressure of 0.05 bar, and pore size of $0.22 \mu \mathrm{m}, \mathrm{b}$ ) flow rate of $0.095 \mathrm{~m} / \mathrm{s}$, pressure of $5 \mathrm{bar}$, and pore size of $0.22 \mu \mathrm{m}$, c) flow rate of $0.533 \mathrm{~m} / \mathrm{s}$, pressure of $5 \mathrm{bar}$, and pore size of $0.22 \mu \mathrm{m}$.

\section{Conclusion}

The findings of this study indicated that the prediction of pomegranate juice permeate flux using the proposed modified fuzzy model was in consistent with the experimental data obtained in the laboratory and low value of error parameters was a clue to consider the Fuzzy Inference System (FIS) as an appropriate model to predict the permeate flux in the membrane clarification process. The proposed model was able to successfully learn the relationship between the input and output parameters. The modified fuzzy model predicted permeates flux during the filtration with MSE of 0.0075 and 0.0085 for MCE and PVDF membranes, respectively. The proposed method can be used in juice production industries and research centers since it is useful to predict and control the permeate flux before the clarification process. The most advantage of the fuzzy modelling is that the concept can be easily understood and it is very flexible especially in food engineering problems. Mathematical concepts which derive a fuzzy model are very simple and the thing that distinguishes this method from other machine learning techniques is its natural reasoning, not inaccessible complexity.

\section{Financial disclosure}

The authors declared no financial interest.

\section{References}

1. Tehranifar A, Zarei M, Nemati Z, Esfandiyari B, Vazifeshenas MR. "Investigation of physico-chemical properties and antioxidant activity of twenty Iranian pomegranate (Punica granatum L.) cultivars", Scientia Horticulturae 2010; 126(2): 180-185.

2. Lansky EP, Newman RA. "Punica granatum (pomegranate) and its potential for prevention and treatment of inflammation and cancer", Journal of Ethnopharmacology, 2007, 109(2), 177-206.

3. Laorko A, Li Z, Tongchitpakdee S, Chantachum S, Youravong W. "Effect of membrane property and operating conditions on phytochemical properties and permeate flux during clarification of pineapple juice", Journal of Food Engineering, 2010, 100(3), 514-521.

4. Aydiner C, Demir, I., Yildiz, E., "Modeling of flux decline in crossflow microfiltration using neural networks: the case of phosphate removal", Journal of membrane science, 2005, 248(1), 53-62.

5. Khayet M, Cojocaru C, Essalhi M. "Artificial neural network modeling and response surface methodology of desalination by reverse osmosis", Journal of Membrane Science, 2011, 368(1), 202-214.

6. Asefpour Vakilian K, Massah J. "An artificial neural network approach to identify fungal diseases of cucumber (Cucumis sativus L.) plants using digital image processing", Archives Of Phytopathology And Plant Protection, 2013, 46(13), 1580-1588.

7. Asefpour Vakilian K, Massah J. "An apple grading system according to European fruit quality standards using Gabor filter and artificial neural networks", Scientific Study and Research: Chemistry and Chemical Engineering, Biotechnology, Food Industry, 2016, 17(1), 75-85.

8. Rai P, Majumdar GC, DasGupta S., De S. "Modeling the performance of batch ultrafiltration of synthetic fruit juice and mosambi juice using artificial neural network", Journal of food engineering, 2005, 71(3), 273-281.

9. Razavi MA, Mortazavi A, Mousavi M. “Application of neural networks for crossflow milk ultrafiltration 
simulation", International Dairy Journal, 2004, 14(1), 69-80.

10. Guadix A, Zapata JE, Almecija MC, Guadix EM. "Predicting the flux decline in milk cross-flow ceramic ultrafiltration by artificial neural networks", Desalination, 2010, 250(3), 1118-1120.

11. Nourbakhsh H, Emam-Djomeh Z, Omid M, Mirsaeedghazi H, Moini S. "Prediction of red plum juice permeate flux during membrane processing with ANN optimized using RSM", Computers and Electronics in Agriculture, 2014, 102, 1-9.

12. Yen J, Langari R.. "Fuzzy logic: intelligence, control, and information", Prentice-Hall, Inc, 1998.

13. Jang JSR. "ANFIS: adaptive-network-based fuzzy inference system", Systems, Man and Cybernetics, IEEE Transactions on, 1993, 23(3), 665-685.

14. Mehraban Sangatash M, Mohebbi M, Shahidi F, Kamyad AV, Rohani MQ. "Application of fuzzy logic to classify raw milk based on qualitative properties", International journal of AgriScience, 2012, 2(12), 1168-1178.

15. Ebadati HR., Vahaji N. "A review on applications of fuzzy logic in food engineering", In 21th Congress on food science and engineering, Shiraz, Iran, 2013.

16. Harris J. "Raw milk grading using fuzzy logic", International journal of dairy technology, 1998, 51(2), 52-56.

17. Mazloumzadeh SM, Shamsi M, Nezamabadi-PourH. "Fuzzy logic to classify date palm trees based on some physical properties related to precision agriculture", Precision agriculture, 2010, 11(3), 258-273.

18. Da Silva IN, Razuk, PC, Flauzino RA. “Applying fuzzy logic systems in the estimation of crossflow filtration processes", In IFSA World Congress and 20th NAFIPS International Conference, 2001, 2740-2745.

19. Cavero D, Tölle KH, Buxadé C, Krieter J. "Mastitis detection in dairy cows by application of fuzzy logic", Livestock Science, 2006, 105(1), 207-213.

20. Prasad K, Nath N. "Comparison of sugarcane juice based beverage optimizations using response surface methodology with Fuzzy method", Sugar Tech, 2002, 4(3-4), 109-115.
21. Jaya S, Das H. "Sensory evaluation of mango drinks using fuzzy logic", Journal of sensory studies, 2003, 18(2), 163-176.

22. Sun, J., Qi, W. M., “Application of Fuzzy Mathematics on Sensory Evaluation of Different Processed Ginger Wine", China Condiment, 2013, 4(9).

23. Yang, L. L., "Fuzzy evaluations in mulberry wine production", IEEE 10th International Conference on Fuzzy Systems and Knowledge Discovery (FSKD), 2013, 369-373.

24. Yazdanshenas, M., Tabatabaee-Nezhad, S. A. R., Soltanieh, M., Roostaazad, R., Khoshfetrat, A. B., "Contribution of fouling and gel polarization during ultrafiltration of raw apple juice at industrial scale", Desalination, 2010, 258(1), 194-200.

25. Razi B, Aroujalian A, Raisi A, Fathizadeh M. "Clarification of tomato juice by cross flow microfiltration", International Journal of Food Science \& Technology, 2011, 46(1), 138-145.

26. Cassano A, Conidi C, Drioli E. "Clarification and concentration of pomegranate juice (Punica granatum L.) using membrane processes", Journal of Food Engineering, 2011, 107(3), 366-373.

27. Pagliero C, Ochoa NA, Marchese J. "Orange juice clarification by microfiltration: Effect of operational variables on membrane fouling", Latin American applied research, 2011, 41(3), 279-284.

28. Mirsaeedghazi H, Emam Djomeh Z, Mousavi SM, Ahmadkhaniha R, Shafiee A. "Effect of membrane clarification on the physicochemical properties of pomegranate juice", International journal of food science \& technology, 2010, 45(7), 1457-1463.

29. Espamer L, Pagliero C, Ochoa A, Marchese J. "Clarification of lemon juice using membrane process", Desalination, 2006, 200(1), 565-567.

30. Soleimani R, Shoushtari NA, Mirza B, Salahi A. "Experimental investigation, modeling and optimization of membrane separation using artificial neural network and multi-objective optimization using genetic algorithm", Chemical Engineering Research and Design, 2013, 91(5), 883-903. 